\title{
Classification of MR Brain tumors with Deep Plain and Residual Feed forward CNNs through Transfer learning
}

\author{
Anilkumar B, P.Rajesh Kumar
}

\begin{abstract}
Medical imaging plays an important role in the diagnosis of some critical diseases and further treatment process of patients. Brain is a central and most complex structure in the human body that works with billions of cells, which controls all other organ functioning. Brain tumours observed as uncontrolled abnormal cell growth in brain tissues. Classification of such cells in a early stage will increase the survival rate of the patient. Machine learning algorithms have contributed much in automation of such tasks. Further improvement in prediction rate is possible through deep learning models. In this paper presents experiments by deep transfer learning models on publicly available dataset for Brain tumour classification. Pre-trained plain and residual feed forward models such as Alexnet, VGG19, ResNet50, ResNet101 and GoogleNet are used for the purpose of feature extraction, Fully connected layers and softmax layer for classification is used commonly. The evaluation metrics Accuracy, Sensitivity, Specificity and F1-Score were computed.

Index Terms: Brain Tumor, Classification, CNNs, Transfer Learning, .
\end{abstract}

\section{INTRODUCTION}

Brain diseases are one of the deadly diseases of peoples [1], like Alzheimer's disease, glioma, meningio, pituitary gioma and so on. Early stage identification of Brain diseases can help in the proper planning of treatment patterns and to improve the survival rate or life span of patients. Computer Aided Diagnosis (CAD) systems are used for medical imaging to diagnose the diseases, MRI is a most popular imaging technique to diagnose the brain of humans without much radiation. MRI gives better resolution of soft tissues. The difference in opinions of doctors and radiologists on the imaging results makes a confusion state for a diagnosis process. The rapid development in artificial intelligence, several automatic detection and diagnosis research contributions are made. The general process to in computer aided diagnosis is starting with pre-processing, feature extraction and reduction and Classification. A brain tumor through MRI images can be detected with traditional feature

Revised Manuscript Received on October 30, 2019.

* Correspondence Author

Anilkumar B*, Assistant Professor, Department of ECE, GMR Institute of Technology, Rajam,India.

Dr.P.Rajesh Kumar, Professor, Department of ECE, University College of Engineering (A), Andhra University, Visakhapatnam, India.

(c) The Authors. Published by Blue Eyes Intelligence Engineering and Sciences Publication (BEIESP). This is an open access article under the CC BY-NC-ND license (http://creativecommons.org/licenses/by-nc-nd/4.0/) extraction procedures such as Local Binary Patterns (LBP) and histogram features extraction [2]. Deep learning has gained more popularity with considering low, medium and high level features from medical imaging data in learning and training processes [3]. CNNs showing better performance in feature extraction than conventional handcrafted feature extraction methods.

Deep learning models are more efficient when applied on a huge volume of training sets. As compared to natural scene images, in medical imaging, such types of huge volume datasets are usually not available. Feature extraction is a very important task in MR brain tumour classification. So more focus ought taken while performing this step in classification. Unlike medical images, a huge volume of image databases like ImageNet, which consist of more than 10 million images [4]. The trained CNNs for ImageNet with perfect knowledge on weights can be used in feature extraction purpose in medical image processing. This transfer of knowledge in feature extraction is through transfer learning is a common practice to classify other images.

There are right now three noteworthy procedures that effectively utilize CNNs to medical image classification. The first procedure is to design and train the "CNN from scratch" [5], Second procedure is to use the pre-trained CNNs for feature extraction rather than the prevailing hand-crafted image features [6] and identification of lung nodule in CT Images [7] and the last procedure is to use pre-trained models by fine-tuning for medical images [8]. In this work BRATS 2017 dataset is used for classification, in this High Grade Gliomas(HGG) and Low Grade Gliomas (LGG) are provided separately. Deep learning models can be plain and residual feed forward CNNs.

\section{A. Plain Feed Forward CNNs}

The plain feed forward neural networks data consistently moves in one forward direction only. The data moves forwarded output layer through hidden layer from input layer, there is no feedback. The popular examples of feed forward networks in deep learning models are Alexnet, VGG16 and VGG19. 


\section{B. Residual Feed Forward CNNs}

Unlike plain feed forward networks, in Residual neural networks the data skip connections or jump over some layers. ResNet models normally skip one or two layers may be ReLu layer, data normalization layers. The popular examples of residual networks in deep learning models are Resnet50, Resnet101, googlenet and so on.

In this work, we make use of the strong suit of deep learning models in an inclusive range of human Brain-related diseases. In Section 2, Discussed the related work for brain tumour classification and other applications. Section 3 dedicated to The Proposed approaches with pre trained CNNs like Alexnet, VGG, ResNet for ImageNet Challenge. Section 4 is for the results and discussions followed by Conclusion.

\section{RELATED WORK}

Wide variety of applications are using deep learning and it's not requires any features which are to be selected by manual or handcrafted [9]. Deep learning algorithms will consider all the features with low and high level sematic information and classify automatically. Transfer Learning targets to gain the knowledge from one or more other fields and improve learning in the target field [10].

Festra method is implemented using transfer learning for microscopic image classification [11]. This method is used for feature extraction and differentiates thin sections of the images. The transfer learning models are compared the CNNs from scratch for plankton classification for a large database. But training CNNs from scratch requires large datasets. Pang et al used a transfer learning approach and a deep learning model based on the basic picture elements of real medical images using supervised training [12]. Alwyn Mathew et al used pre-trained Google's inception model for ATM surveillance dataset for security purpose [13]. For an image search and classifications, The pre-trained CNNs, like VGG-Net and AlexNet are used to extract the features [14]. Meng, Dan, et al used a fully connected network (FCNet) using VGGNet and a deep classifier for liver fibrosis classification [15]. Dimitrios Marmanis et al proposed a simple and computationally efficient approach to schema to combine features extracted from deep networks [16].

\section{CLASSIFICATION THROUGH TRANSFER LEARNING}

\section{A. DataSet}

The BRATS dataset [17] MR brain nifty format image dataset used for classification, in this High Grade Gliomas(HGG) and Low Grade Gliomas are provided separately with ground truth for segmentation.

LGG and HGG volumetric information (3D) are converted into 2D using ITK SNAP tool [18]. MR brain volumetric information can be viewed in three modes as Axial, coronal and sagittal. In this work Axial mode 2D slices are taken from FLAIR modality Images with reference to the ground truth 2D Slices. Each image is with dimensions 240X240X155 and Slices are extracted randomly between 70th and 110th Axial Mode. We have extracted 500 slices from Benign (LGG), 500 slices from Malignant (HGG) and totally 1000 slices of both as Brain Tumour Data Set (BTDS). The resultant extracted the 2D slices are with size $240 \mathrm{X} 240$.

The BTDS spited into Training (70\%), Development (20\%) and Testing (10\%) sets. Below table represents the number of slices for each Training, Validation and Testing sets respectively.

Table. I: Training, Validation and Testing Data Split up

\begin{tabular}{|c|c|c|c|c|}
\hline & BTDS & Training & Validation & Testing \\
\hline $\begin{array}{c}\text { No. of } \\
\text { Images }\end{array}$ & 1000 & 700 & 200 & 100 \\
\hline Benign & 500 & 350 & 100 & 50 \\
\hline $\begin{array}{c}\text { Malignan } \\
\mathbf{t}\end{array}$ & 500 & 350 & 100 & 50 \\
\hline
\end{tabular}

\section{B. Pre-Processing}

Pre-processing of BTDS is an important step in advance to the training, validation and testing phases of model on images. This step avoids over fitting problem by removing the noise, removing bias effects and performing data augmentation on the dataset.

\section{Denoising}

For Denoising the input MR image slices, a feed forward Denoising Convolution Neural Network (DnCNN) and median filter is used. This DnCNN can handle Gaussian Denoising with unknown noise level. This can also detect the simple nosie level and other high frequency artifacts of images. DnCNN not only removes the noise from the images but also benefited by GPU computing.

\section{Data Augmentation}

Data Augmentation is the major part of pre-processing in transfer learning. This involves many techniques as Resizing, Scaling, Translation, Rotation, Flipping, Adding Salt and Pepper noise, Lightening Conditions, Perspective Transform

Amongst data augmentation techniques, the specific operation can be preferred and applied to the images based on the requirement. Different pre-trained images accept diverse RGB image sizes at input layers. All the training, validation and testing images should be resized as per requirements of the pre trained network. So, at present, Image resizing is the only augmentation technique that is going to be applied. Table 2 shows the augmentation required for different pre-trained networks.

Table. II: Data augmentation for different pre-trained networks

\begin{tabular}{|c|c|}
\hline $\begin{array}{c}\text { Image size in } \\
\text { BTDS }\end{array}$ & $\begin{array}{c}\text { Resize of Images as per } \\
\text { pre-trained Model }\end{array}$ \\
\hline 240X240 & Alexnet / 227X227 \\
\hline 240X240 & VGG19 / 224X224 \\
\hline 240X240 & Resnet50 / 224X224 \\
\hline 240X240 & Resnet101/ 224X224 \\
\hline 240X240 & Googlenet/ 224X224 \\
\hline
\end{tabular}

Sample Training MR Brain Axial slices are shown IN Fig.2

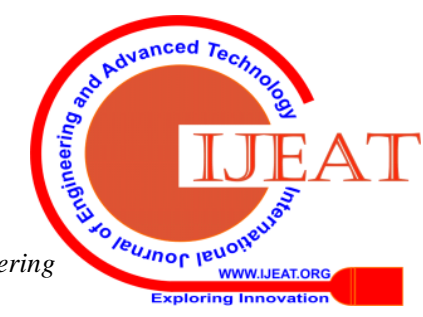




\section{TRANSFER LEARNING MODEL}

Figure 2 shows the transfer learning model for different pre-trained Deep learning models. Through this model we have trained models like Alexnet, VGG19, Resnet50, Resnet101, etc...

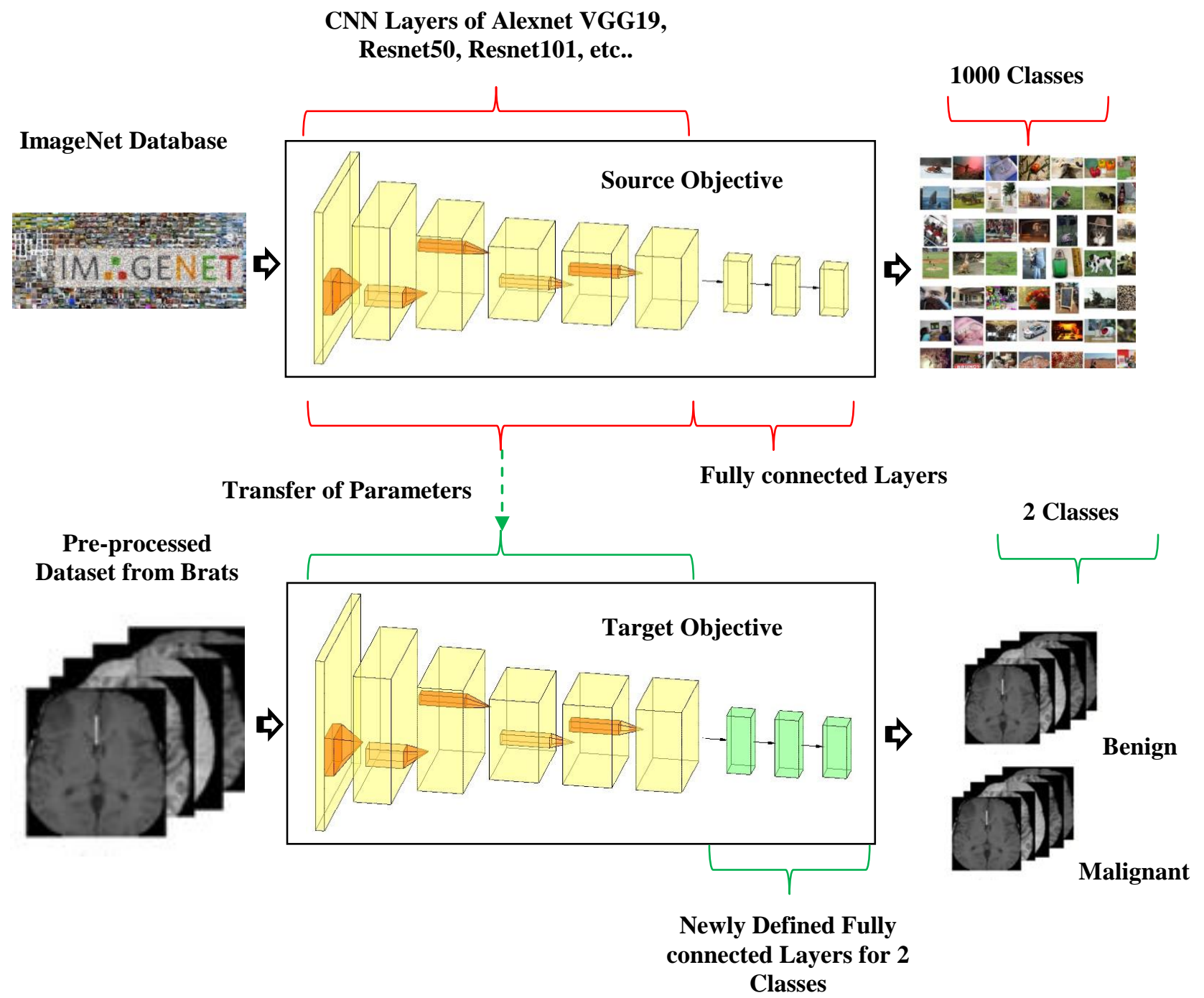

.Fig.1. Transfer Learning Model 

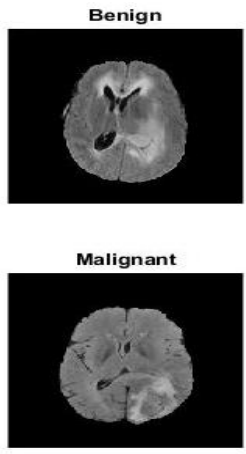

Benign

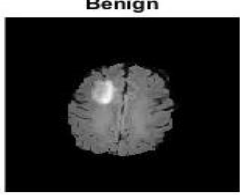

Benign

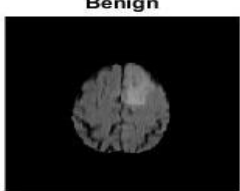

Malignan

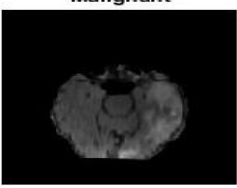

Benign

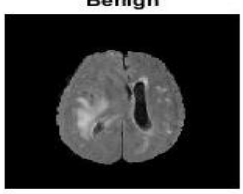

Malignan

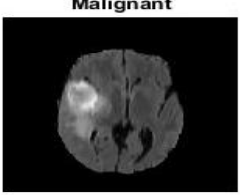

Benign

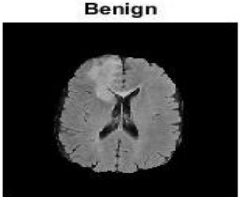

Benign

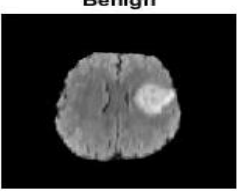

Benign

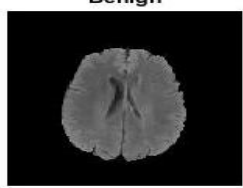

Malignan

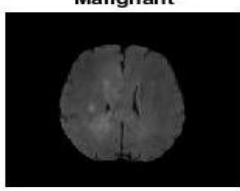

Malignant

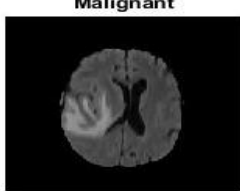

Fig.2. Sample training MR Brain Image Axial Slices

. All the pre-trained models used for classifying 1000 classes. Their parameters (weights) are trained to classify approximately 1000 classes of scene images. In this work all these models are retrained for 2 classes (Benign and malignant) by freezing convolution layers and redefining the number of classifying classes.

\section{RESULTS AND DISCUSSIONS}

\section{A. Training and Validation Phases}

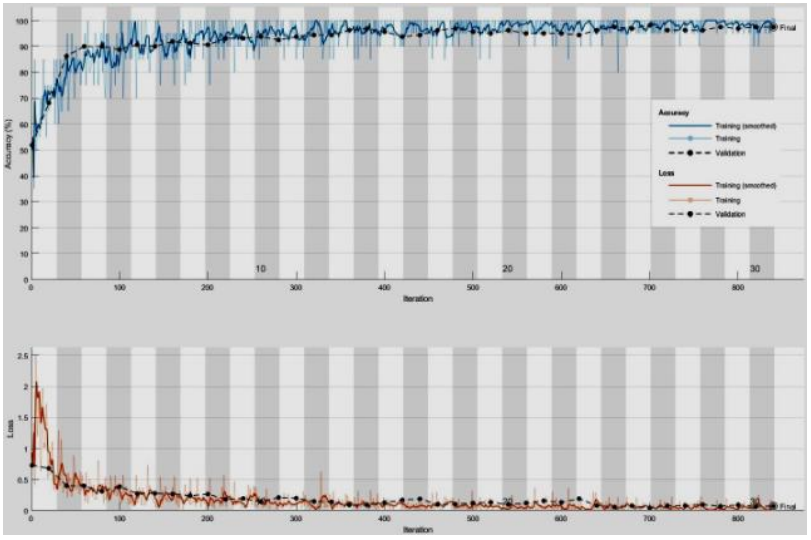

Fig.3. TL_Alexnet Training and Validation

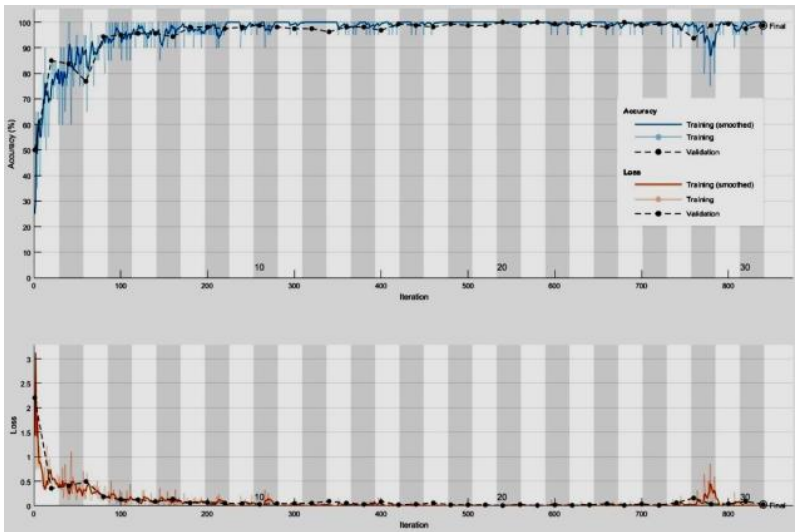

Fig.4. TL_VGG16 Training and Validation

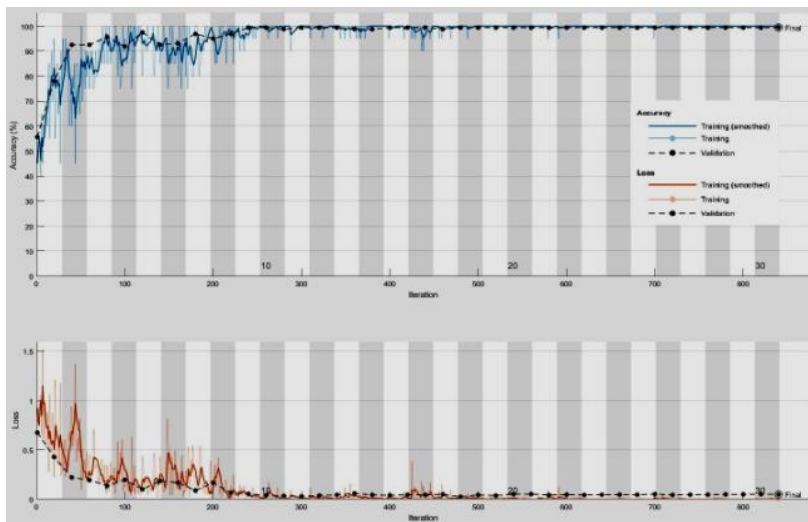

Fig.5. TL_VGG19 Training and Validation

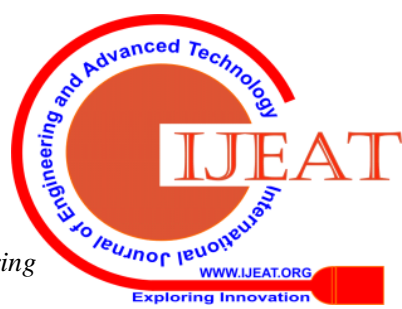




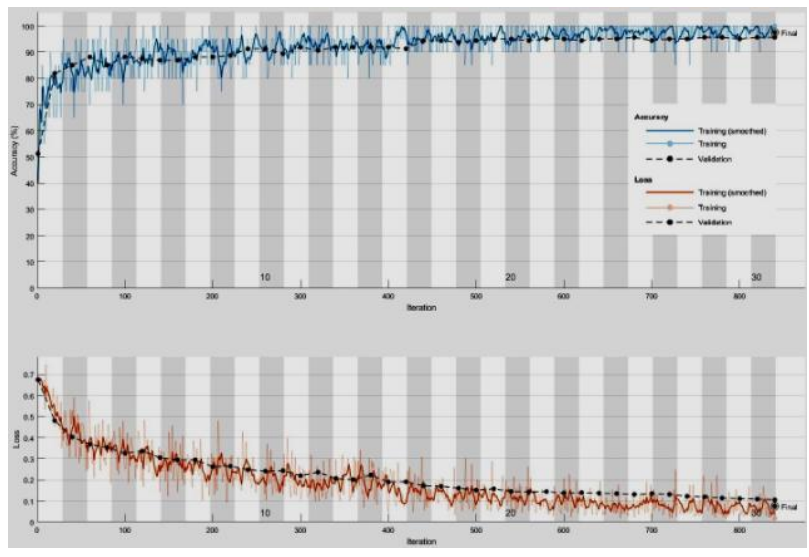

Fig.6. TL_resnet50 Training and Validation

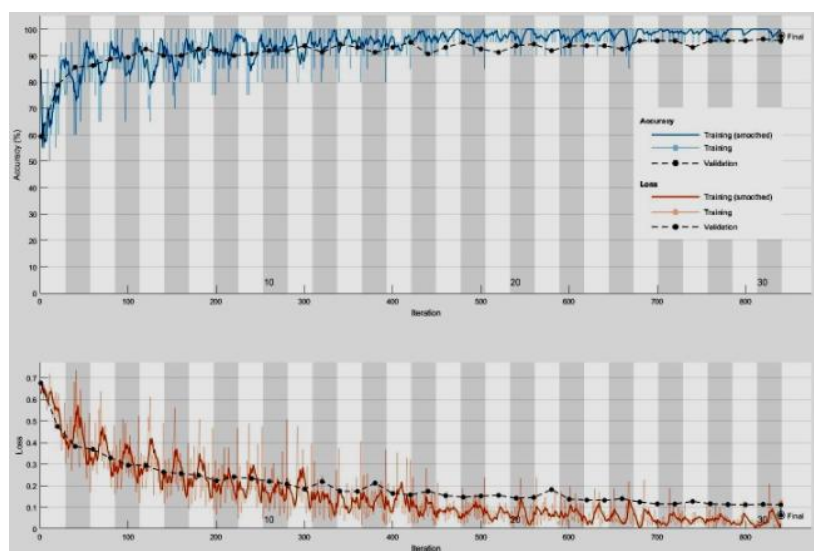

Fig.7. TL_resnet101 Training and Validation

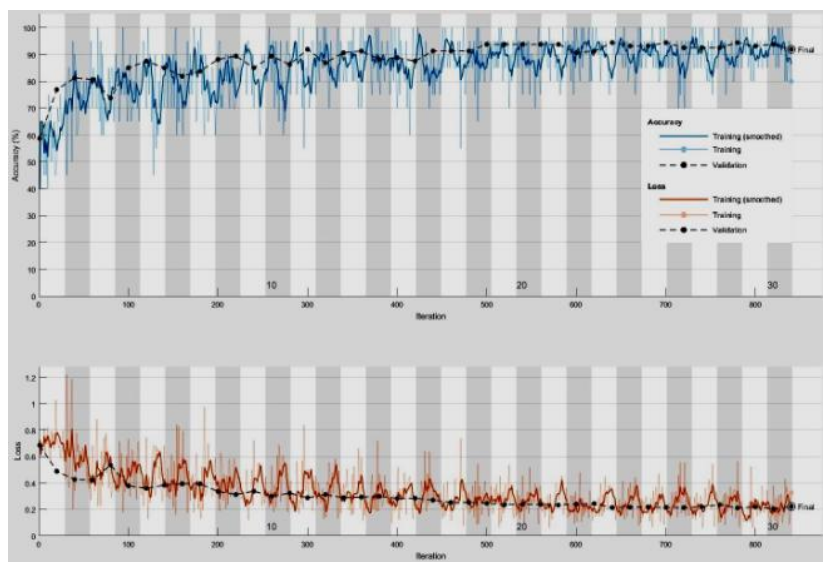

Fig.8. TL_googlenet Training and Validation

Fig.3 to Fig.8 shows training and validation phases of pre-trained models. Each model has taken different timing for completing these phases. Table III shows the details of time taken for training and validation by each model.
Table. III Data augmentation for different pre-trained networks

\begin{tabular}{|c|c|}
\hline $\begin{array}{c}\text { Resize of Images } \\
\text { as per pre-trained } \\
\text { Model }\end{array}$ & $\begin{array}{c}\text { Time taken to train the Model } \\
\text { (hours }: \text { minutes }: \text { seconds) }\end{array}$ \\
\hline Alexnet & $00: 02: 10$ \\
\hline VGG19 & $00: 28: 11$ \\
\hline Resnet50 & $00: 07: 18$ \\
\hline Resnet101 & $00: 22: 47$ \\
\hline Googlenet & $00: 02: 34$ \\
\hline
\end{tabular}

\section{B. Confusion Matrix and Validation Metrics}

The performance of the classification model is often described by 'confusion matrix'. The elements of confusion matrix are as folllows

True Positives(TP): No. of Benign/ Malignant MR images those are classified as they are Benign/ Malignant.

True Negatives(TN): No. of Non-Benign/ Non-Malignant MR images those are classified as they are Non-Benign/ Non-Malignant.

False Positives(FP): No. of Non-Benign/ Non-Malignant images those are classified as they are Benign/ Malignant. False Negatives(TN): No. of Benign/ Malignant MR images those are classified as they are Non-Benign/ Non-Malignant. Validation Metrics:

$$
\begin{aligned}
\text { Accuracy } & =\frac{T P+T N}{T P+T N+F P+F N} \\
\text { Sensitivity } & =\frac{T P}{T P+F N}
\end{aligned}
$$

Specificity $=\frac{T N}{T N+F P}$

$P P V=\frac{T P}{T P+F P}$

$F 1-$ Score $=2 \cdot \frac{P P V \times T P R}{P P V+T P R}$ 


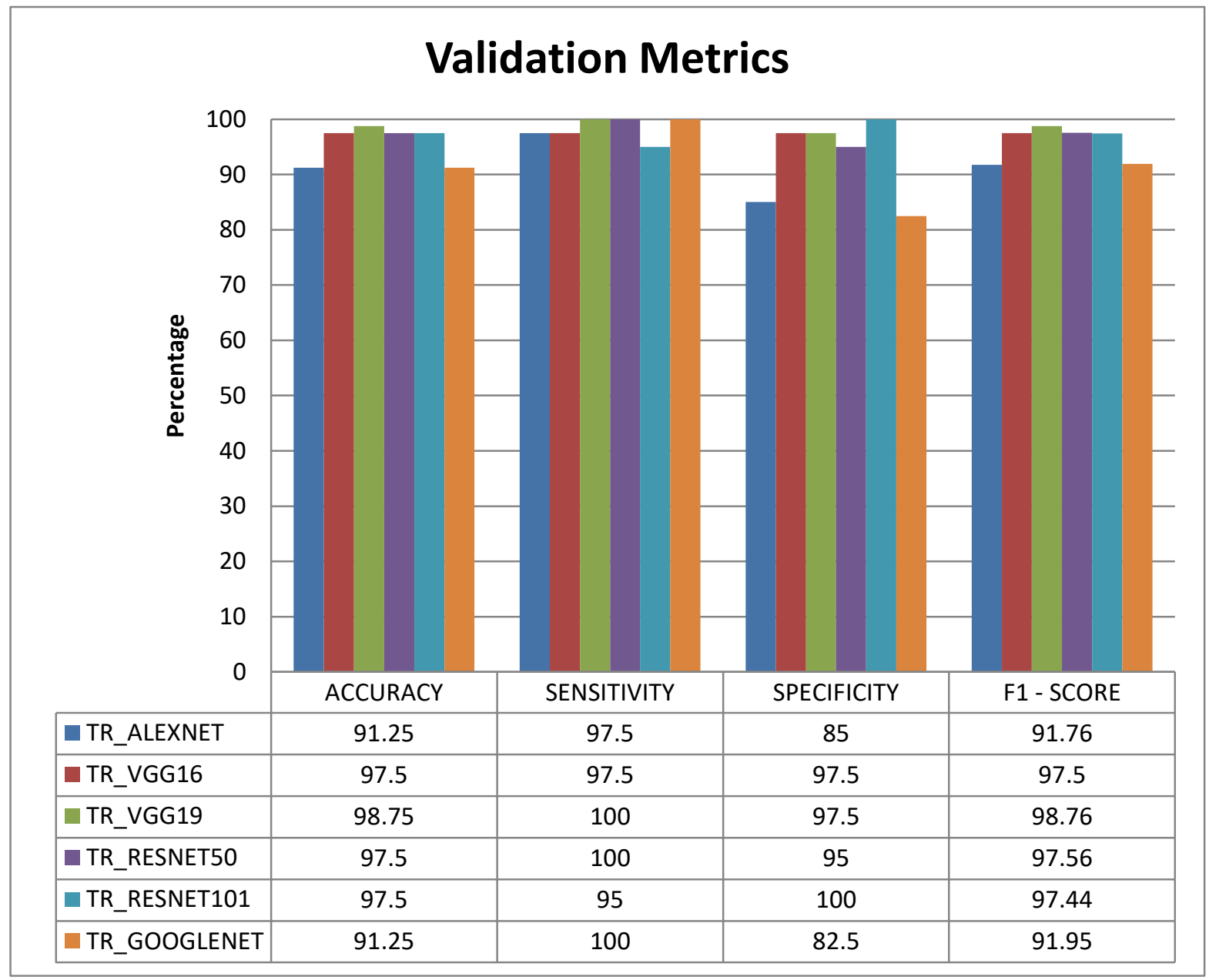

Fig.10. Comparison of pre-trained Deep learning models on BTDS

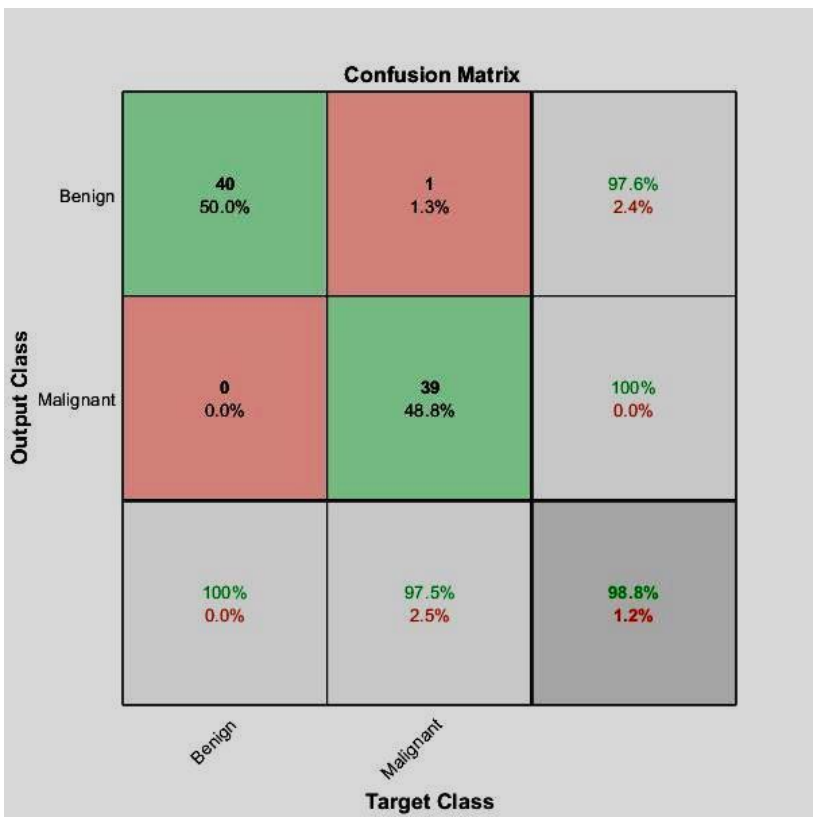

Fig.9. Confusion Matrix for VGG19

\section{CONCLUSION}

In this paper, transfer learning based approach for brain tumour image classification is implemented. The state of art pre-trained plain and residual feed forward CNNs, like Alexnet, VGG16, VGG19, ResNet50, ResNet101 and Google net are used, All these networks are trained on BTDS prepared to classify Benign and Malignant tumors. The same model can be used to classify other organs of the human body with other medical imaging techniques as well. Performance Metrics are evaluated, VGG19 has shown better accuracy as 98.75\% among the other deep learning models.

\section{REFERENCES}

1. R.J. Dawe, et al., Postmortem brain MRI is related to cognitive decline,independent of cerebral vessel disease in older adults, Neurobiol. Aging 69(2018) 177-184.

2. Fletcher-Heath, Lynn M., et al. "Automatic segmentation of non-enhancing brain tumors in magnetic resonance images." Artificial intelligence in medicine 21.1 (2001): 43-63.

3. Y. LeCun, K. Kavukcuoglu, and C. Farabet, "Convolutional networks and applications in vision," Proceedings of IEEE International Symposium on Circuits and Systems (ISCAS), pp. 253-256, 2010.

4. A. Krizhevsky, I. Sutskever, and G. E. Hinton, "Imagenet classification with deep convolutional neural networks," in NIPS, 2012.

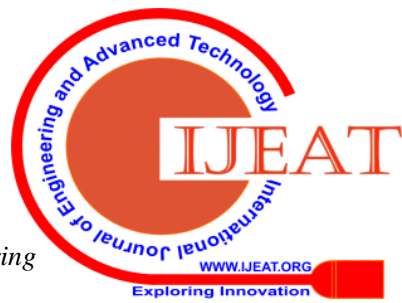


5. B. Menze, M. Reyes, and K. Van Leemput, "The multimodal braintumor image segmentation benchmark (BRATS)," IEEE Trans. Med.Imag., vol. 34, no. 10, pp. 1993-2024, Oct. 2015.

6. Y. Bar, I. Diamant, H. Greenspan, and L. Wolf, "Chest pathology detection using deep learning with non-medical training," Proc. IEEE 12th Int. Symp. Biomed. Imag., pp. 294-297, 2015.

7. B. van Ginneken, A. Setio, C. Jacobs, and F. Ciompi, "Off-the-shelf convolutional neural network features for pulmonary nodule detection in computed tomography scans," Proc. IEEE 12th Int. Symp. Biomed. Imag., pp. 286-289, Apr. 2015.

8. T. Schlegl, J. Ofner, and G. Langs, "Unsupervised pre-training across image domains improves lung tissue classification," in Medical Comput. Vision: Algorithms for Big Data. New York: Springer, 2014, pp. 82-93.

9. Greenspan, Hayit, Bram Van Ginneken, and Ronald M. Summers. "Guest editorial deep learning in medical imaging: Overview and future promise of an exciting new technique." IEEE Transactions on Medical Imaging 35.5 (2016): 1153-1159.

10. M. Long, J. Wang, and M.I. Jordan, Deep Transfer Learning with Joint Adapta- tion Networks, arXiv preprint arXiv:1605.06636. 2016.

11. Li, Na, et al. "A transfer learning method for automatic identification of sandstone microscopic images." Computers \& Geosciences 103 (2017): 111-121.

12. Pang, Shuchao, Zhezhou Yu, and Mehmet A. Orgun. "A novel end-to-end classifier using domain transferred deep convolutional neural networks for biomedical images." Computer methods and programs in biomedicine 140 (2017): 283-293.

13. Mathew, Alwyn, et al. "An Improved Transfer learning Approach for Intrusion Detection." Procedia computer science115 (2017): 251-257.

14. L. Zheng, Y. Zhao, S. Wang, J. Wang, and Q. Tian, "Good practice in cnn feature transfer," vol. abs/1604.00133, (2016). [Online]. Available: http://arxiv.org/abs/1604.00133

15. Meng, Dan, et al. "Liver fibrosis classification based on transfer learning and FCNet for ultrasound images." Ieee Access 5 (2017): 5804-5810.

16. Marmanis, Dimitrios, et al. "Deep learning earth observation classification using ImageNet pretrained networks." IEEE Geoscience and Remote Sensing Letters 13.1 (2015): 105-109.

17. Menze, Bjoern H., et al. "The multimodal brain tumor image segmentation benchmark (BRATS)." IEEE transactions on medical imaging 34.10 (2014): 1993-2024.

18. Yushkevich, Paul A., and Guido Gerig. "ITK-SNAP: an intractive medical image segmentation tool to meet the need for expert-guided segmentation of complex medical images." IEEE pulse 8.4 (2017): 54-57.

\section{Authors Profile}

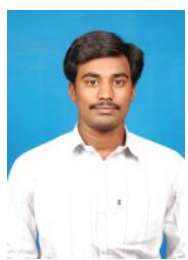

B. Anil kumar, completed Bachelor of Technology from JNTU, Hyderabad in 2005 and completed Master of Technology with Embedded systems specialization from JNTU, Hyderabad in 2008. He is working as an Assistant Professor in the department of ECE, GMRIT-Rajam, India and pursuing $\mathrm{PhD}$ (Part-time) in Medical Image analysis from Andhra University, Visakhapatnam, India.

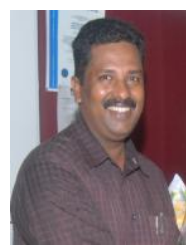

Dr. P. Rajesh Kumar, completed Bachelor of Engineering OU in 1992 and completed Master of Engineering from AU in 1995. He received Ph.D from AU in 2006 and currently working as a Professor and BOS chairman, Department of ECE, College of engineering (A), Andhra University, Visakhapatnam., India. 